\title{
Development of Innovative Secured Remote Sensor Water Quality Monitoring \& Management System: Case of Pangani Water Basin
}

\author{
Kaizilege Mwemezi ${ }^{a}$ (Research Scholar), Anael Sam ${ }^{a}$ (Senior Lecturer) \\ ${ }^{a}$ Department of Communications Science and Engineering, Nelson Mandela African Institution of Science and \\ Technology, Arusha, Tanzania.
}

Received: 19 September 2018; Accepted: 17 October 2018; Published: 08 January 2019

\begin{abstract}
Wireless sensor networks (WSN), when applied to the field of water quality monitoring and management, has the potential to bring numerous benefits as compared to traditional methods since it is accurate, reliable in remote areas and in the tough condition such as during rainy seasons, and less costly. With the ever-growing application of Internet of Things (IoT) and technology in general, sensor devices have become less expensive and widely used in many applications that require remote monitoring. In this work, we present an innovative and secure water monitoring and management system using remote sensor prototype, which has been developed to monitor physiochemical parameters including $\mathrm{pH}$, Turbidity, Temperature, and Dissolved Oxygen. Qualitative research methods were used for gathering system requirements through questionnaires and interviews conducted in Pangani water basin authority, in the United Republic of Tanzania. Documents were also reviewed to provide secondary knowledge. Furthermore, the system was developed using Dynamic System Development Methodology (DSDM). Firstly, the proposed system prototype is able to provide real-time measurements accurately. Secondly, the proposed system uses Advanced Encryption Standards to provide a secure transmission and storage of data transmitted from remote sensor nodes to a central database system. Thirdly, the web application was developed for data visualization using tabular and graphical formats. Lastly, the system prototype provides decisions support on quality of water present in Pangani river basin by evaluating sensor measurements and sends SMS alerts once the measured value is above the recommended standard values.
\end{abstract}

Index Terms: Water quality, Raspberry Pi, pH sensor, Turbidity sensor, Temperature sensor, Dissolved Oxygen sensor, Pangani River basin, Remote sensor.

(C) 2019 Published by MECS Publisher. Selection and/or peer review under responsibility of the Research Association of Modern Education and Computer Science.

\footnotetext{
* Corresponding author. Tel.: +255753280921
}

E-mail address: mwemezik@nm-aist.ac.tz 


\section{Introduction}

The decline of water quality in rivers caused by toxic chemicals, domestic wastes, and unnecessary nutrients, which results from the combination of human activities has become an ever-growing problem worldwide [1]. As outlined by many researchers, industrial activities, agriculture activities, and population growth are the leading drivers in polluting water sources $[2,3]$. Consequently, the contamination of water sources caused by the mentioned activities has caused a major concern in the health and environment of the involved individuals and the general public. The alarming rates of waterborne disease cases and scarcity of clean and quality water sources has caused many countries around the world to collaborate in programs that will ensure sustainable water management through an integrated approach in order to minimize the problems caused by poor water quality [4].

In the United Republic of Tanzania, the Ministry of Water and Irrigation (MoWI) through Water Sector Development Programme (WSDP 2006-2025) is responsible to ensure that water sources including rivers, dams, lakes, and groundwater are properly managed and monitored by providing the necessary resources. For instance, Water Basin Authorities (WBA) under the MoWI have established the network of monitoring stations mainly in rivers to monitor meteorological, quality and quantity aspects of water in the river basins. As a result, the measurements obtained from stations have become a crucial part in the assessment and protection of water sources in the basins [5].

However, the main challenges, faced by water basin authorities is lack of modern and sophisticated equipment to measure physical, chemical and biological water parameters in a timely, accurate and, reliable manner [6]. The present water monitoring systems in the basins are divided into two categories, which are manual systems and data loggers. The process of monitoring water quality parameters through manual systems involves collection of water samples from various stations in the river basins and sending them to a laboratory where each sample is measured and results are obtained and recorded. On the other hand, Data loggers installed in various stations record data, which are mainly water level and meteorological data whereby the experts from the WBA collect them through USB flash drive for assessment and analysis. Both systems prove to be inefficient since it takes a long time to obtain sample results, and it is also costly in terms of manpower and finances required to facilitate the monitoring process. In addition, the storage and security of data collected from the stations are threatened since data are stored in manual books and files [7,8].

Over recent years, Wireless Sensor Networks (WSN) have been used to monitor the quality of water in the river basins worldwide, since they prove to be a more efficient method as compared to manual systems. Furthermore, studies have shown that WSN offers many important features, which can be entirely used to provide a faultless monitoring process. For instance, Hu et al. [9] designed a water quality monitoring system using Zigbee based wireless sensor to measure $\mathrm{pH}$, temperature, and turbidity. The system was able to provide data in real-time with low cost in terms of installation and maintenance. However, the system has low processing speed and memory, which can lead to continuous system failure. Vijayakumar \& Ramya [10] also developed a real-time monitoring system for water quality in the Internet of Things (IoT) environment to measure $\mathrm{pH}$, turbidity, conductivity, temperature, and dissolved oxygen. However, their system doesn't have security features and information distribution points such as alarming messages, which can help the authorities to take required actions timely. Likewise, Faustine et al. [11] designed a wireless sensor network prototype for monitoring water quality in the Lake Victoria Basin (LVB). The system was designed to measure $\mathrm{pH}$ level, temperature, Electrical Conductivity, Dissolved Oxygen and transmit data to a server in the office. Data stored in the server are displayed in both tabular and graphical form using web application. However, authors recommended that further studies should be made on lowering power consumption of the system so as to prolong the battery life, and the use of Zigbee module has a benefit of lowering power consumption at the expense of low data rate and short data transmission range.

Therefore, the aim of this paper is to present an Innovative Water Quality Monitoring System using Remote 
Sensors (IWQMSRS) which will be used to measure pH, Turbidity, Dissolved Oxygen (DO) and Temperature in upper Pangani river basin. The system developed consists of four parts. The first part is data acquisition, whereby a Raspberry Pi 3 model B was used as a controller integrated with a $\mathrm{pH}$ sensor, Turbidity sensor, Temperature sensor, and DO sensor. The second part is a network module with GSM/WCDMA/LTE capacity that provides a network connection to a controller through IEEE 802.11.g standards and an internet connection, which is used to transfer data to a web server in the office. The third part is a MySQL database server, which is used to store data collected from the sensing units. The fourth part is a data presentation and web application unit, which is used to display data in the graphical and tabular view. The proposed system ensures a remote access to data acquisition unit for configurations and status checks through the use of Secure Shell (SSH) and Video Network Connectivity (VNC). Apart from that, the proposed system ensures a secure transmission of data from the data acquisition unit to a data post-processing unit through the use of Advanced Encryption standards (AES) encryption and decryption algorithms. Furthermore, to ensure reliability of the system save the measurements from the sensing unit once there is no network connectivity. Moreover, the proposed system has a decision support module which evaluates the measurements from water quality sensors and sends SMS alerts to officials responsible for monitoring river basin.

\section{Materials and Methods}

\subsection{Data collection}

The process of data collection was facilitated using interviews and documents review for the aim of gathering system requirements. To achieve this, the interviews were conducted three times at Pangani Water Basin Authority (PWBA) covering Environmental department, Planning Department, Information and Communication Technology (ICT) department, and Water Quality department. The results from the interviews provided themes, which were used to set up the qualitative analysis on documents review. To achieve that, a total of 70 research papers from journals and institutional reports were selected. The QDA Lite analysis tool was used through the textual analysis module to gather both functional and non-functional requirements.

\subsection{System Design and Development}

The development of the entire system prototype was guided by using the Dynamic System Development Method (DSDM). This was because of the following reasons: It emphasizes on user and developer collaboration, development of the system is incremental and entirely driven by user needs, and encouraging frequent delivery and testing of working software [12]. In the web application development, the Model View Controller (MVC) architecture was adopted because of the following criteria: MVC is very useful for development of software which involves extensive programming; it includes security features which are appended to applications automatically during development, it is easier to debug and test applications in MVC than other traditional approaches [13]. Furthermore, Unified Modelling Language (UML) was adopted during database design, while the flowchart diagram was adopted during the design of programs specifically for reading data from sensors but also sending them to the database. The database was developed to run on MySQL database server. On the other hand, OrCAD capture tool was used for making hardware circuit for design purpose.

\section{System Requirements}

The data collected from interviews, questionnaires, and documents review were used as a key factor in describing clearly services and constraints that should be enclosed in the system. To achieve this, both services and constraints are divided into functional and non-functional requirements as presented in Table 1. 
Table 1. System Requirements

\begin{tabular}{ll}
\hline System Requirements (FR) & Descriptions \\
\hline $\begin{array}{l}\text { The system shall monitor automatically } \mathrm{pH}, \\
\text { Turbidity, DO, Temperature }\end{array}$ & The data required by the users of the system should be real-time and updated.
\end{tabular}

Officials from the basin are required to login into the system.

The system shall display data in graphical form.

The system shall display data in tabular form.

The system shall calculate the average values of measured parameters and display the results after a specified time

The system shall redistribute information timely to specified officers once parameters exceeded a standard value.

Security

Accessibility

Usability, Reliability, and Portability
The system should be used only by registered by officials from the Pangani Water Basin Authority

The system shall provide data in graphical format: $\mathrm{pH}$ (y-axis) vs time (x-axis), Turbidity (y-axis) vs time (x-axis), DO (y-axis) vs time (x-axis), Temperature (y-axis) vs time (x-axis).

The system shall provide data in tabular format with the inclusion of the following attributes: site name, site ID, sensor ID, location, $\mathrm{pH}$, turbidity, $\mathrm{DO}$, and temperature, time and date.

The system shall calculate the average values of $\mathrm{pH}$, temperature, $\mathrm{DO}$, and turbidity and display the results.

The system shall send alert SMS to officers when sensors detect that the accepted values of either $\mathrm{pH}$, turbidity, $\mathrm{DO}$ or temperature have been violated.

The system shall be secure since the measurements taken in the stations are only required to be disclosed by MoWI which is responsible according to the laws of the country. Moreover, only authorized users are permitted to access and use the system

The system shall be accessed at any time. Since the system will be installed in remote places that are basically far from the central offices then the use of a mobile network for communication and transmission of data is required. Also, high penetration of mobile network even in remote places has led to propose a system that shall be incorporated with mobile network interface.

Users with different levels of computer literacy shall be able to operate easily the proposed system. Therefore, both user interfaces and system operation shall be designed in such a way that it can be manipulated easily by its users. Users with different levels of computer literacy shall be able to operate easily the proposed system. Therefore, both user interfaces and system operation shall be designed in such a way that it can be manipulated easily by its users.

\section{Proposed System Architecture Design}

The process of developing a system prototype for monitoring water quality consists of four basic modules, which are data acquisition, data transmission and encryption, data storage, and data visualization and decision support module. To achieve the development of each module, both hardware and software were integrated. As seen in Fig.1. 


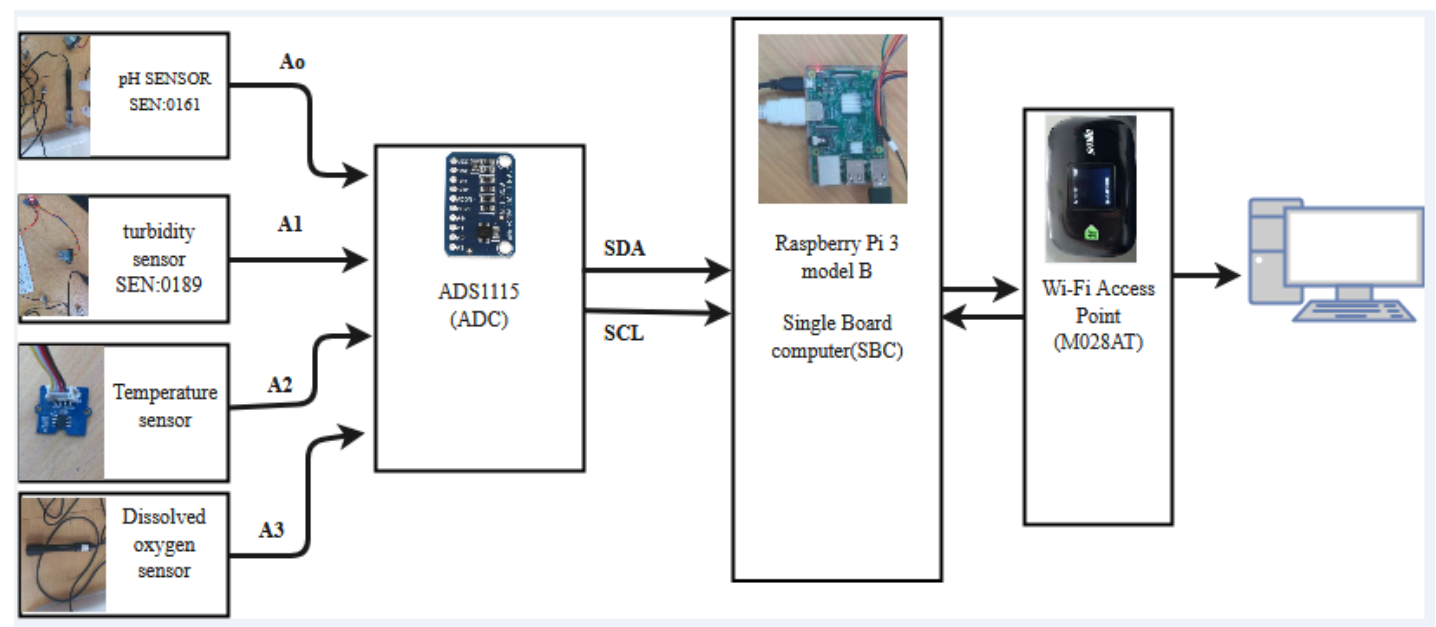

Fig.1. Block Diagram for Proposed System Architecture

\subsection{Data Acquisition unit Components}

Based on the system requirements, the development of Data Acquisition Unit (DAU) consists of five components: a single board computer, a pH sensor, a turbidity sensor, a temperature sensor, a dissolved oxygen sensor, and an ADC board. The factors that lead to the selection of each component involved for a design of DAU are presented in the subsection 5.1.1, 5.1.2, 5.1.3, 5.1.4, 5.1.5, and 5.1.6; and summarized in Table 2.

\subsubsection{Single Board Computer (SBC)}

The Raspberry Pi 3 model B was selected among other SBCs to be used for development of DAU by considering many important features it possesses. Some of its features are identified for proper development are: it uses python as a core programing language hence brings simplicity, scalability, portability, and richness of libraries [14]; It has a processing speed of $1.4 \mathrm{GHz}$; memory (RAM) $1 \mathrm{~GB}$; supports external storage up to 32GB (micro SD); low power consumption 5V, 2A; and less expensive (\$35). Furthermore, it can be accessed remotely using SSH and VCN interfaces thus simplify its operation. Table 2. presents the comparison of Raspberry Pi 3 model B with Nucleo-F410re and ODROID-XU4 boards [15,16,17].

Table 1. Comparison of SBCs

\begin{tabular}{llll}
\hline & Raspberry Pi 3 model B & Nucleo-F410re & ODROID-XU4 \\
\hline Power & $5 \mathrm{~V}, 3.3 \mathrm{~V}, 2 \mathrm{~A}$ (grid and battery) & $5 \mathrm{~V}, 800 \mathrm{~mA}$ & $5 \mathrm{~V}, 4 \mathrm{~A}$ \\
Microcontroller & ARM quad-core & STM-32-ARM & Samsung Cortex \\
Cost & Low cost & Low cost & High cost \\
Memory & 1GB & $512 \mathrm{~KB}$ & 2GB \\
Support & High & Low & Low
\end{tabular}

\subsection{2. $p H$ Sensor Module}

SKU: SEN0161 is an analog pH sensor module with high response time (1min) and a capacity to measure $\mathrm{pH}$ in all range (0-14). The SKU: SEN0161 sensor provides the output with high accuracy of $\pm 0.1 \mathrm{pH}$ and 
operates with an input voltage of 5VDC, hence it easily integrated with both Raspberry Pi 3 model B and ADS1115 boards. Moreover, it is a low-cost device with a vast technical support freely available on the internet. Therefore, the aforementioned factors led to the selection of SKU: SEN0161 for the prototype development [18].

\subsubsection{Turbidity Sensor Module}

SKU: SEN0189 is an analog/digital turbidity sensor module, which uses Nephelometric principle to measure turbidity of water and provide output with high accuracy and high response time (500ms). It expresses the measurements results in terms of Nephelometric Turbidity Unit (NTU) and it has the ability to operate in the temperature range of $5^{\circ} \mathrm{C}-90^{\circ} \mathrm{C}$. Furthermore, the SKU: SEN0189 uses 5VDC and 40mA input voltage and input current respectively for its operations. It is less expensive and easily available in the market [18]. All these led to the choice of SKU: SEN0189 for the prototype development

\subsubsection{Dissolved Oxygen Sensor Module (EZO-DO)}

EZO-DO is a family of Atlas sensors for measuring dissolved oxygen (DO) in water using a probe which contains Polytetrafluoroethylene membrane, cathode, and anode whereby oxygen molecules are diffused into membrane and measured. It is a well-calibrated sensor and provides output measurements with high accuracy of about $+/-0.05 \mathrm{mg} / \mathrm{L}$. It has a response time of 1 second and a capacity to measure a range of $0.01-100 \mathrm{mg} / \mathrm{L}$ amount of DO in water. The EZO-DO supports two data protocols I2C and UART, which can be easily interfaced with Raspberry Pi model B SBC. It's operating voltage and current is either $5 \mathrm{~V} / 13.5 \mathrm{~mA}$ or $3.3 \mathrm{~V} / 12.1 \mathrm{~mA}$. Therefore, the reasons mentioned above led to the selection of EZO-DO as a perfect sensor for the proposed system [19].

\subsubsection{Grove-Temperature sensor V 1.2 module}

Grove Temperature V1.2 is an analog sensor module, which use thermistors to measure the ambient temperature. It provides output measurements with an accuracy of $+/-1.5^{\circ} \mathrm{C}$ and operates within the temperature range of $-40^{\circ} \mathrm{C}$ to $+125^{\circ} \mathrm{C}$. Its operating voltage is $3.3 \mathrm{~V}-5 \mathrm{~V}$, zero resistance of $100 \mathrm{~K}$, and Nominal B constant of $4250 \mathrm{~K}-4299 \mathrm{~K}$. The Grove Temperature V1.2 is easy to calibrate and it is supported by many platforms including Arduino, Raspberry Pi, Beaglebone, Wio, Linkltone It is for the reasons mentioned above that Grove-Temperature sensor V1.2 was selected as the perfect sensor for the proposed system[20].

Table 2. Selected Sensor Modules

\begin{tabular}{lllll}
\hline & $\mathrm{pH}$ sensor & Turbidity sensor & DO sensor & Temperature sensor \\
\hline Model & SEN0161 & SEN0189 & EZO-DO & Grove Temp V1.2 \\
Operating voltage & $5 \mathrm{~V}$ & $5 \mathrm{~V}$ & $3.3-5 \mathrm{~V}$ & $3.3-5 \mathrm{~V}$ \\
Accuracy & \pm 0.1 & \pm 0.5 & \pm 0.05 & \pm 1.5 \\
Cost & low & Low & low & low \\
Response & $1 \mathrm{~min}$ & $500 \mathrm{~ms}$ & $1 \mathrm{~s}$ & $1 \mathrm{~s}$
\end{tabular}

\subsubsection{Analog to Digital Converter Board (ADC)-ADS1115}

ADS1115 is a chip used to convert analog signals to digital signals. It has high precision since it provides 16bit with 800 samples/second over I2C data protocol. The ADS1115 chip has 4 input channels, which make it easy to accommodate 4 analog sensors in one chip. In addition, it uses a programmable amplifier with up to 
16x, which makes it easier to amplify signals with smaller values. The chip operates with the voltage range from $2 \mathrm{~V}-5 \mathrm{~V}$ and current of $150 \mu \mathrm{A}$. Also, it is easy to configure and interface with controllers. Among other ADC, the ADS1115 is identified as suitable to the proposed system [21].

\subsubsection{Connections of DAU Hardware Components}

SKU: SEN0169 pH sensor module is made up of 5-pins ( $\mathrm{V}+, \mathrm{G}, \mathrm{G}, \mathrm{P}$, and Po). $\mathrm{V}+$ and $\mathrm{G}$ are connected to GPIO 5V pin and Ground (GND) of Raspberry Pi 3 model B respectively. The Po pin is connected to A1 pin of the ADS1115. The ADS1115 is supplied with a 5V pin of Raspberry Pi 3 model B and the same applied to GND pin. The I2C protocol pins SCL and SDA of ADS1115 are connected to SCL (GPIO2) and SDA (GPIO3) pin of Raspberry Pi 3 model B. The SKU: SEN0189 turbidity sensor module consists of 4 pins (G, A, D, V). The V-pin is connected to 5V supply voltage, G-pin is connected to GND pin, A-pin is connected to A2 of ADS1115.The EZO-DO -Dissolved Oxygen sensor module consists of 6 pins (GND, TX/SDA, RX/SCL, Vcc, $\mathrm{PRB}$, and PGND). The Vcc pin is connected to Voltage supply pin-5V and GND-pin is connected to GND-pin of Raspberry Pi 3 model B. TX/SDA-pin and RX/SCL-pin is connected to SDA-pin and SCL-pin of Raspberry Pi 3 model B board. The temperature sensor module Glove-Temperature sensor V 1.2 module consists of 4 pins (SIG, NIC, VCC, and GND). The SIG-pin is connected to Ao-pin of ADS1115 while VCC and GND pin is connected to the voltage supply source.

The I2C is interface is configured to allow communication between sensors and Raspberry Pi 3 model B. It is configured under Raspian Operating system through command line interface whereby the ADS1115 board is on address $0 \times 48$ and EZO-DO is on address 0x63.

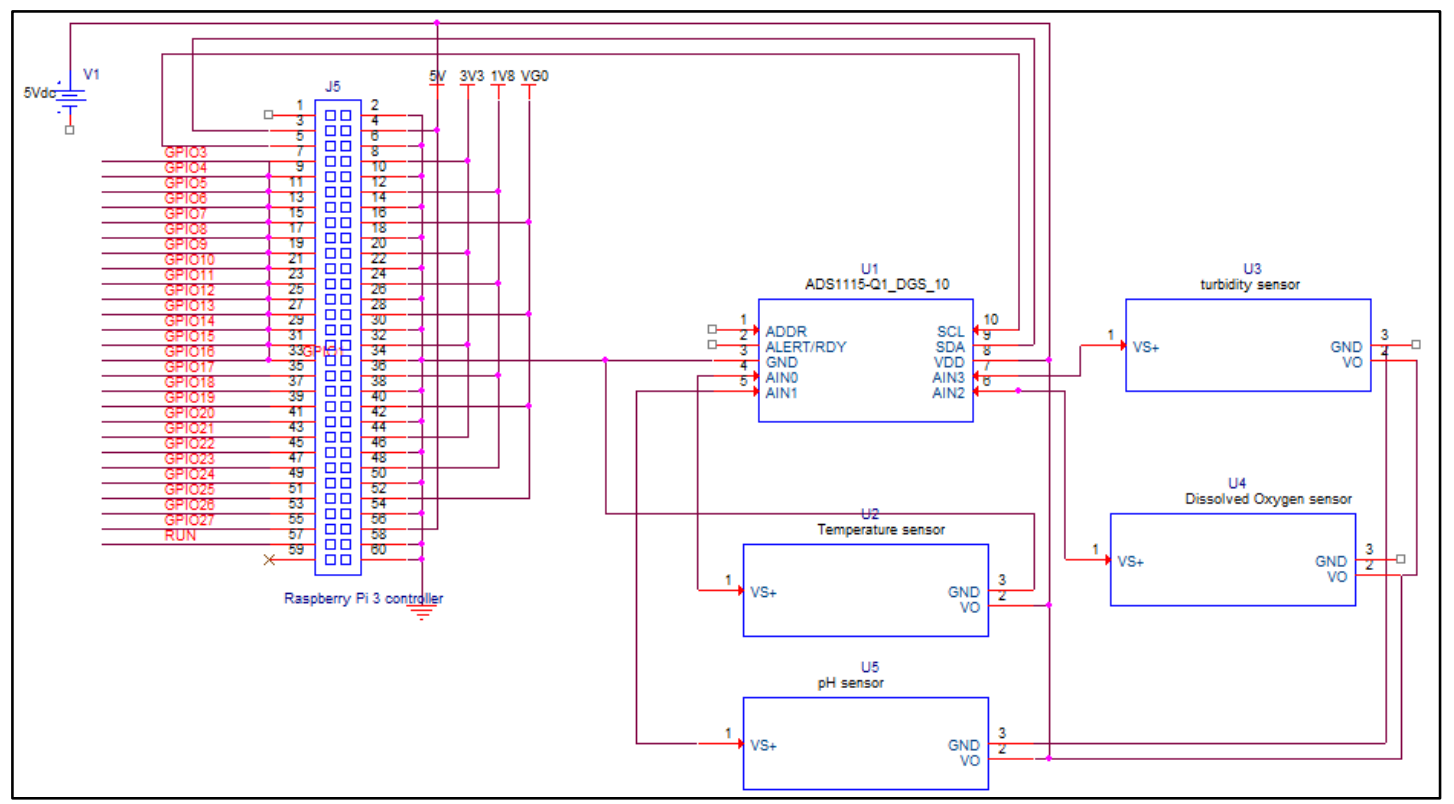

Fig.2. Schematic Diagram for Data Acquisition Unit (DAU).

\subsubsection{Reading and Calibrating Data from Sensors}

The data from sensors are retrieved from Raspberry Pi 3 model B - I2C pins using a program written in python3. The python3 is incorporated within Raspian Operating system. To read data from I2C pins, AdaFruit 
ADS1x15 python library was imported to a program in order to enable the process of reading of digital signals coming from ADS1115 channels. The digital signals from sensors are identified according to the assigned channel name of ADS1115 board. According to the design of this system the channel was assigned as follows: Ao-channel to Temperature sensor, A1-channel to $\mathrm{pH}$ sensor, A2-channel to Turbidity sensor. The measurements retrieved from the channels are expressed in a 16-bit format, which is within the range of -32768 to 32767. Decoding the results to analog value the equation (1) is used as recommended by ADS1115 board datasheet whereby VTB is the analog voltage, VADC is a digital voltage value, and $6.144 \mathrm{~V}$ is the reference voltage set when using a programmed gain of $2 / 3$.

$$
V T B=(V A D C \div 32767) \times 6.144
$$

The calibration of sensors readings was done using both graphical methods and datasheet provided formulas. For that case, the temperature reading was obtained by using mathematical relationship presented on the equation (2), whereby thermistor constant (B), VPH is resistance of temperature sensor at $0^{\circ} \mathrm{c}$ and the voltage reading of $\mathrm{ADC}$ channel and the $\mathrm{pH}$ reading were obtained using graphical methods. Multiple points were collected using six $\mathrm{pH}$ solutions with known values against sensor reading expressed in terms of voltage. The reading of the turbidity sensor was obtained by applying the equation (4), which consists of the relationship between voltage and turbidity values. While the Dissolved Oxygen was obtained through the datasheet provided values.

$$
\text { temperature reading }=1.0 /(\log V T B / B+1 / 298.15)-273.15
$$

$$
p H \text { reading }=-14.3031 V P H+60.3142
$$

$$
\text { turbidity reading }=-90.57 V T B^{2}+123.1 V T B+1020
$$

\section{Data Transmission and Security Unit}

\subsection{Wireless Module M028AT}

In this system, the transfer of sensor measurements from data acquisition unit to a remote database server was facilitated using M028AT wireless module. To achieve that, a network connection was established in two phases. In the first phase, the Raspberry Pi 3 model B SBC was connected to M028AT through IEEE 802.11.g wireless standard. While, in the second phase, the M028AT wireless module was connected to the internet through a cellular network. To access the internet connection through the cellular network, the M028AT uses built-in SIM card module with a capacity to access multiple cellular network technologies such as FDDLTE1800/2100MHz, WCDMA1800MHz/2100MHz, and GSM 900MHz/1800MHz.The M028AT is very useful when used in remote places since it uses a low-power rechargeable battery and it has high transmission range [22].

\subsection{Sensor Measurements Security Module}

The Advanced Encryption Standards (AES) was used to secure sensor measurements transmitted from DAU to a remote web server located in the office. This section describes how AES algorithms are provided and applied to the proposed system to ensure that it provides a secure transmission of data transmitted from remote sensing unit to the database. 


\subsubsection{Description of AES Algorithms}

The AES algorithms were designed by National Institute of Science and Technology (NIST) in the year 2001. It is designed mainly to replace Data Encryption standards. AES is the family of symmetric key algorithms whereby the sender and receiver use the same key for encryption and decryption. These algorithms are complex algorithms which are so difficult for attackers to break. The structure of AES consists of plaintext with the size of 16 bytes, and a key with the size of either 16, 22, or 32 bytes. The input to encryption and decryption is a $4 \times 4$ matrix block of bytes. This $4 \times 4$ matrix block passes through many encryption or decryption states before the output block is produced. Likewise, the key is converted to block of a square matrix whereby it passes to an array of scheduled words before the output is produced. As a result, the ciphertext is produced when the input is passed to encryption states, while the plaintext later is produced when the ciphertext is passed in decryption states[23].

\subsubsection{Implementation of AES in the Proposed System}

In this system, the AES module was written in the Raspberry Pi 3 model B using python programming language. To achieve this, the pycryptodome package was installed in the SBC. The pycryptodome package contains AES encryption algorithms libraries, which are used to facilitate the process of encrypting sensor measurements data. Thereafter, the implementation of the encryption module was as follows: Generating of 16bytes secret key and 16-bytes initialization vector, creating an AES cipher which is used to encrypt the data, converting the sensor measurements from float to a string data type, passing the sensor measurements to AES cipher algorithms to produce ciphertext, creating another ciphertext by concatenating ciphertext and initialization vector, and sending ciphertext to a remote database. Figure 4 shows the flow diagram for AES encryption process.

To convert the measurements to plaintext the AES decryption module was created inside the web application. Again, the pycryptodome package is installed to facilitate the process of decryption. After installing pycryptodome package, the implementation of decryption module was as follows: generating the secret key and initialization vector which resemble to that of encryption module, creating AES decryption algorithms, passing the ciphertext to AES decryption algorithms, getting the plaintext and display the results in a web application.

\section{Web Application Design}

The web application was designed to ensure it provides data storage, data visualization, user authentication, and decision support. To achieve that, the application interacts with three entities to facilitate the process of dataflow. Fig.3. presents a data flow between entities and application. The main application, which is represented as IWQMSRS-APPLICATION is interacting with Data Acquisition unit (sensor node), system administrator, and Users (PWBA- Employee).

The IWQMSRS software consists of a MySQL database on the server side and a user application run on the web browser. Both user application side and server side were developed using Django 2.0.5 framework. A Django framework is a free python web framework which uses MVC approach to develop a web application in a fast, secured, and easy way. As compared to other frameworks, Django is selected due to the following reasons: Firstly, it can be used for both large and small applications. Secondly, it provides a built-in function such as authentication, routing, templating, and database administration. Thirdly, it has many plugins and libraries which is suitable for web application developments in a fast-paced environment [24]. 


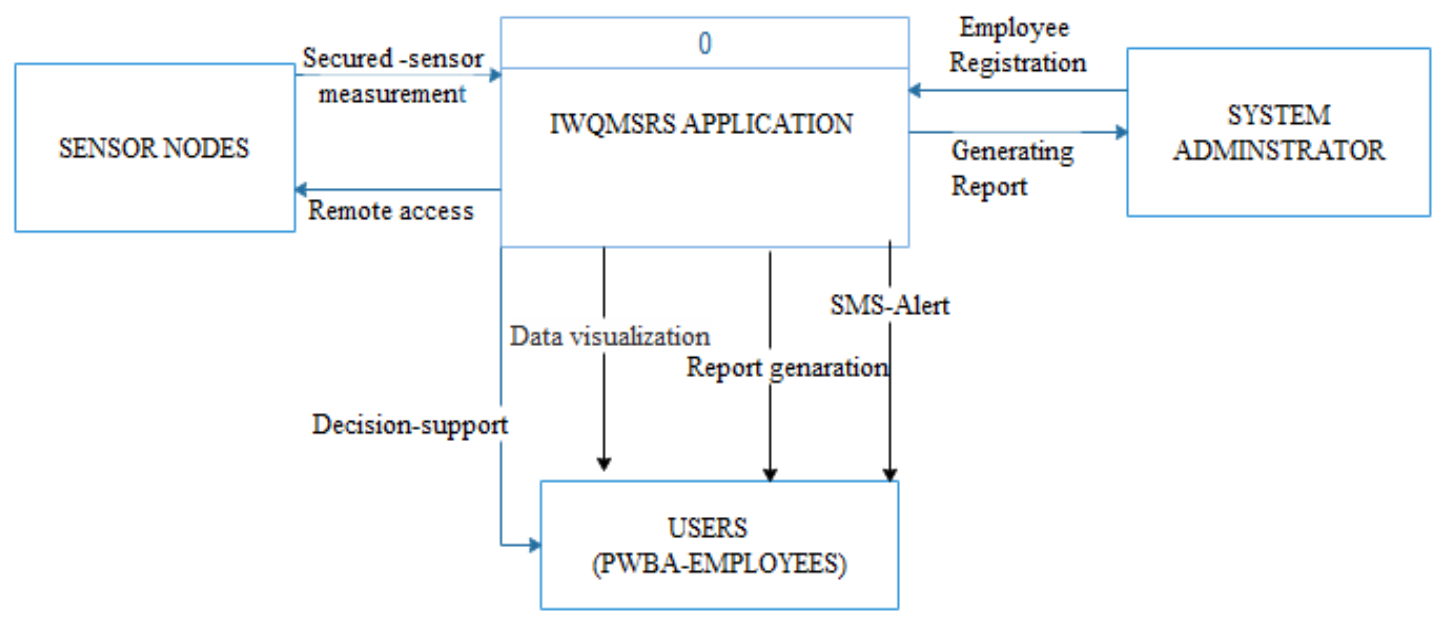

Fig.3. Contextual Diagram for IWQMSRS Application

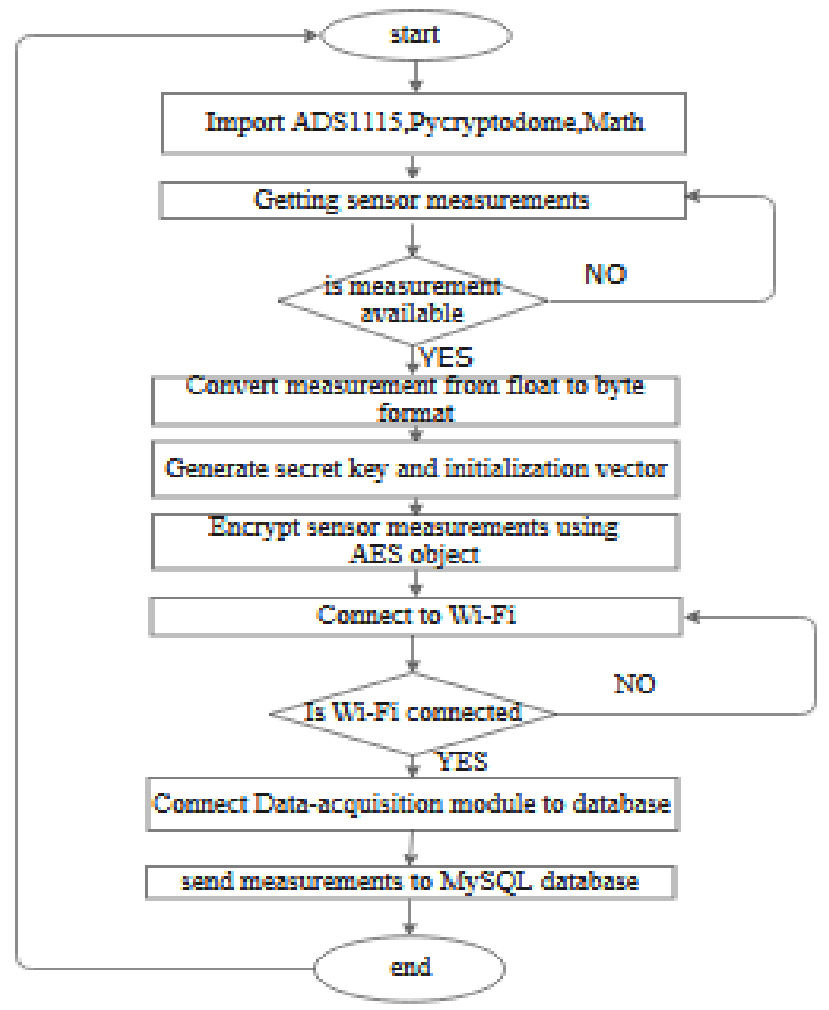

Fig.4. Flowchart Diagram for Sensor Measurements and AES Encryption 
Table 3. Present Comparison between Web Application Developments Frameworks

\begin{tabular}{lll}
\hline \multicolumn{1}{c}{ Django } & \multicolumn{1}{c}{ Flask } & \multicolumn{1}{c}{ Pyramid } \\
\hline $\begin{array}{l}\text { It is used for both small and large } \\
\text { application. }\end{array}$ & It is used for small application. & $\begin{array}{l}\text { It is used both for small and large } \\
\text { application }\end{array}$ \\
$\begin{array}{l}\text { Built-in authentication, routing, } \\
\text { templating, and database administration }\end{array}$ & $\begin{array}{l}\text { No built-in authentication, routing, } \\
\text { templating, and database administration. }\end{array}$ & $\begin{array}{l}\text { Built-in authentication, routing but } \\
\text { uses external templating and database } \\
\text { administration }\end{array}$ \\
Large support & Small support & Medium support
\end{tabular}

Like other MVC framework, Django consists of three main layers, which are View, Model, and Controller layers. The Controller layer is used for processing the logic which originates from the user request and providing the response accordingly. The Model layer is used for structuring and manipulating data used by an application. The View Layer is used for rendering the information from user's requests in the web page [25] The figure 5 presents Django MVC pattern for the IWQMSRS application for the Dashboard fragment, which contains other fragments such as Water Quality Parameter (WQP) Tables, WQP Charts, and WQP Support, that are accessed by clicking the buttons. For instance, to view water quality parameters $(\mathrm{pH}$, Temperature, DO, Turbidity) measurements in table format, a user needs to click WQP table button. When user clicks the button, the URL to WQP Table fragment is mapped to TableView method in the View Layer using Django URL configuration file. Thereafter, the TableView method in the view layer queries the water quality parameters $(\mathrm{pH}$, Temperature, DO, Turbidity) through a Model layer to the database. Thereafter, the query result is stored in the variable present in the TableView method and rendered to WQP Table web page.

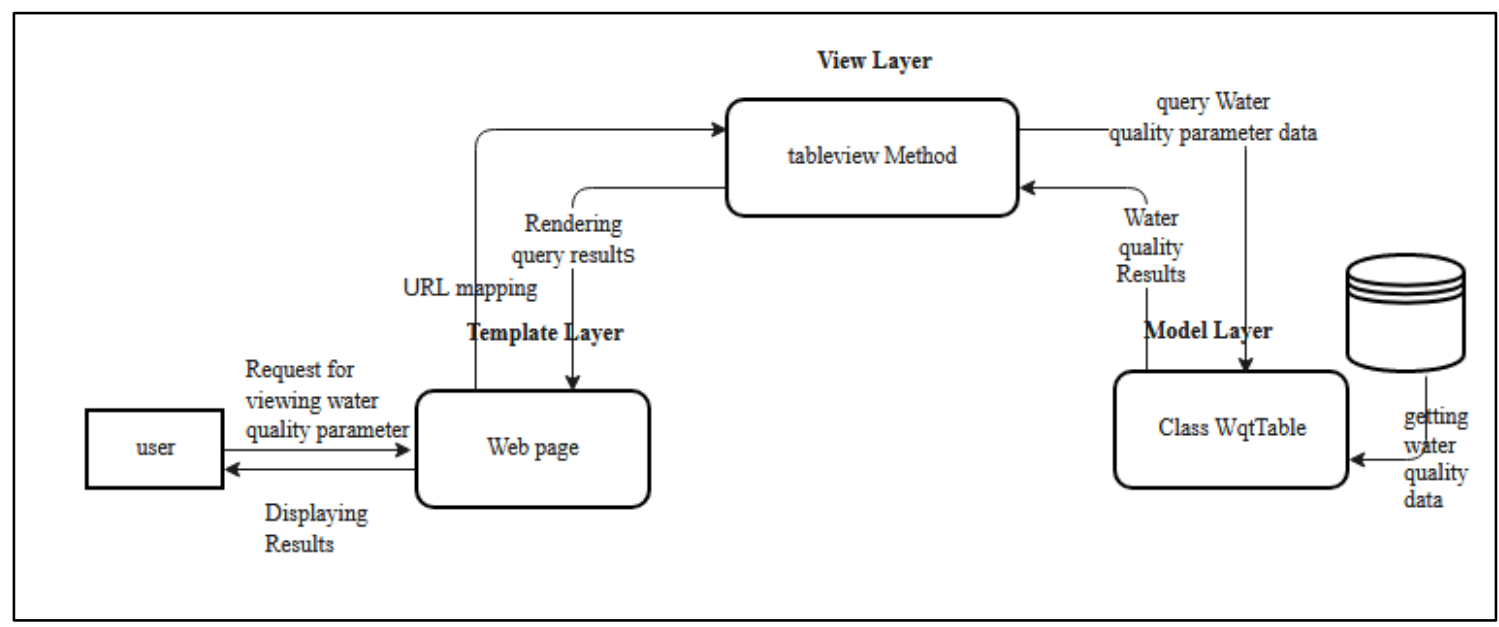

Fig.5. Django MVC Pattern when Accessing WQP in the Table View

\subsection{Database Unit}

The IWQMSRS database was developed to store both users' and application information. To achieve that, the design process started by analyzing the requirements which resulted in the formulation of four classes, attributes, and methods used for data processing. The classes of this system are Sensor, Site, Employee, Employee and Site description. In this system, MySQL database management system was selected because of 
the following factors: it is free and run in many platforms including Windows, Linux, Mac OS; it has large technical support and easily operated. The connection between Django framework and MySQL was done through settings.py file whereby database credentials including Host, Port, User, and Name were clearly defined and set. The Django model.py file was used to create Sensor, Site, Employee, and Employee and Site description classes that were mapped to the database tables. The fields of each class are mapped as table columns.

\subsection{Decision Support Module}

This module was designed purposely to provide the decision on the quality of water in Pangani river basin. It summarizes the WQP data from the database, which receives real-time measurements (pH, Turbidity, DO, temperature) from remote sensors and compares them with pre-defined standards values defined by Tanzania Water Standards [26], as shown in Table 4. Thereafter, the short message service (SMS) alert will be sent to a water site supervisor (PWBA-Employee) for the immediate actions once measurements exceed the standard values.

Table 4. Physiochemical Water Parameters Standard Values

\begin{tabular}{lllll}
\hline & $\mathrm{pH}$ & Dissolved Oxygen & Temperature & Turbidity \\
\hline Standard Values & $6.5-8.5$ & $3-15 \mathrm{mg} / \mathrm{l}$ & $20-35^{\circ} \mathrm{C}$ & $<300 \mathrm{NTU}$
\end{tabular}

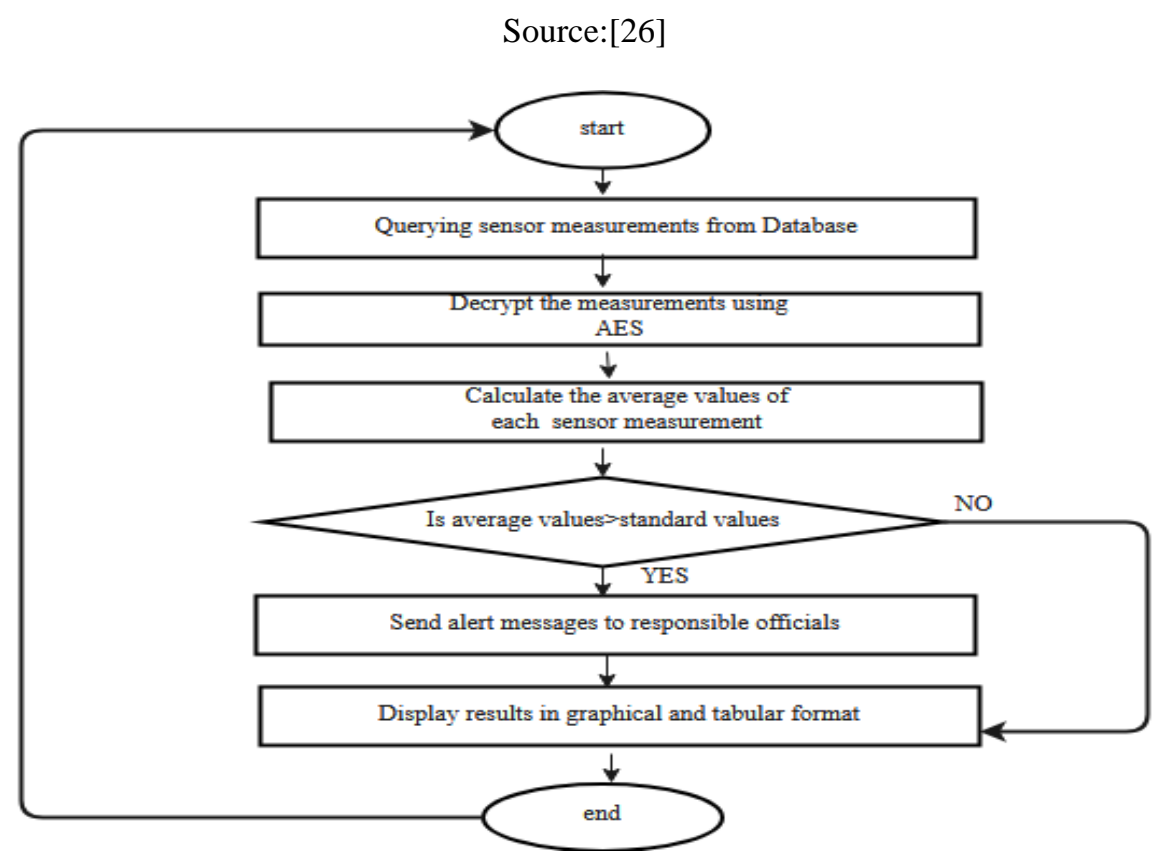

Fig.6. A Flow Chart Diagram for Decision Support Module

The SMS module was developed inside a web application using nexmo-API. The nexmo-API is a set of cloud-based communications services that are designed to reduce the complexity caused by mobile carriers. The choice of SMS cloud-based APIs was based in the following: availability of its services on given location (country) based on mobile phone policies; cost of SMS services; and support provided by the product's 
developers. Due to these factors, nexmo-API was deemed most suitable for the system among other SMS-APIs such as Textmagic and Twilio, nexmo [27].

In this system, nexmo-API was installed and imported to the Django framework through a class named nexmo. A client method from the nexmo class was then set with a secret token and key as input parameters. Thereafter, the alert message was set to be sent to a responsible site supervisor once either of the parameters exceeds the pre-defined standards.

The implementation process of decision support module was as follows: (1) Querying $\mathrm{pH}$, temperature, DO, turbidity measurements from database present on the previous three hours using query slicing techniques present in python list; (2) Evaluating the average values of each parameters provided by the previous process; (3) Comparing the average values of each parameter to pre-defined standard value;(4) Conditionally send the SMS alert to responsible site supervisors once the value of WQP exceed the standard values; (5) Display the results for visualization.

\subsection{System Results Interfaces}

Figure 7 shows a web page interface of encrypted sensor measurements including $\mathrm{pH}$, turbidity, dissolved oxygen, and temperature received from five sample sites. Using Advanced Encryption Standards (AES), these sensor measurements were encrypted in Data Acquisition Unit (DAU) level before transmitted over Wi-Fi and cellular communication (WCDMA) to a central database system (IWQMSRS-database). To ensure security, these measurements were passed over 10-rounds of transformations before it produces a ciphertext. Applying AES encryption standards to the developed prototype ensured prevention of a sensor network attacks such as interruption attacks, interception attacks, fabrication and modification attacks. Both of these attacks may result in false errors message, denial network traffic, and control of information if security measures are not in place.

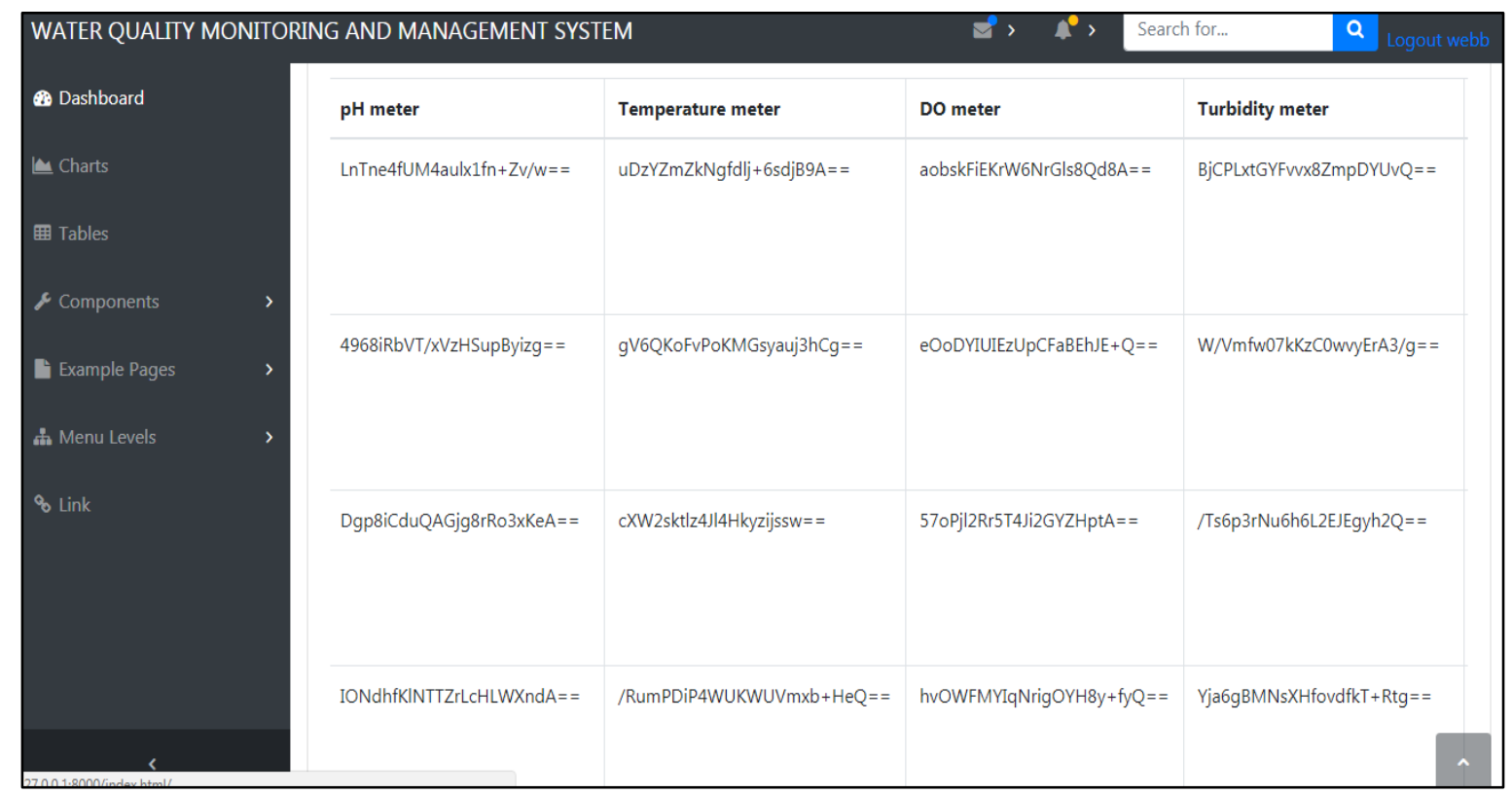

Fig.7. Sensors Measurements in Cipher text Format

Figure 8 and Figure 9 present a web interface of decrypted sensor measurements transmitted from remote 
sensors in tabular and graphical format respectively. These measurements are obtained from Nambala, Tengeru_catchment, Tengeru_Juu, Usa_catchment, and Usa_town sites. The pH values recorded are 6.4 ,6.7, 8.4, and 6.8, DO values recorded $6.0,8.2,14.0,11.2$, and 15.0, for turbidity the recorded measurements are $7.5,41.0,23,24.0$, and 27.0, while water temperature recorded are $23.0,23.67,22.45,21.0$, and 24.45. Both of these measurements are within the standard value of safe water uses except for Tengeru catchment whereby there was an act of feeding livestock during the collection of samples. Furthermore, the results are obtained within a short time duration as opposed to the existing manual systems which take about $6 \mathrm{hrs}$ to complete measurements of five (5) sites.

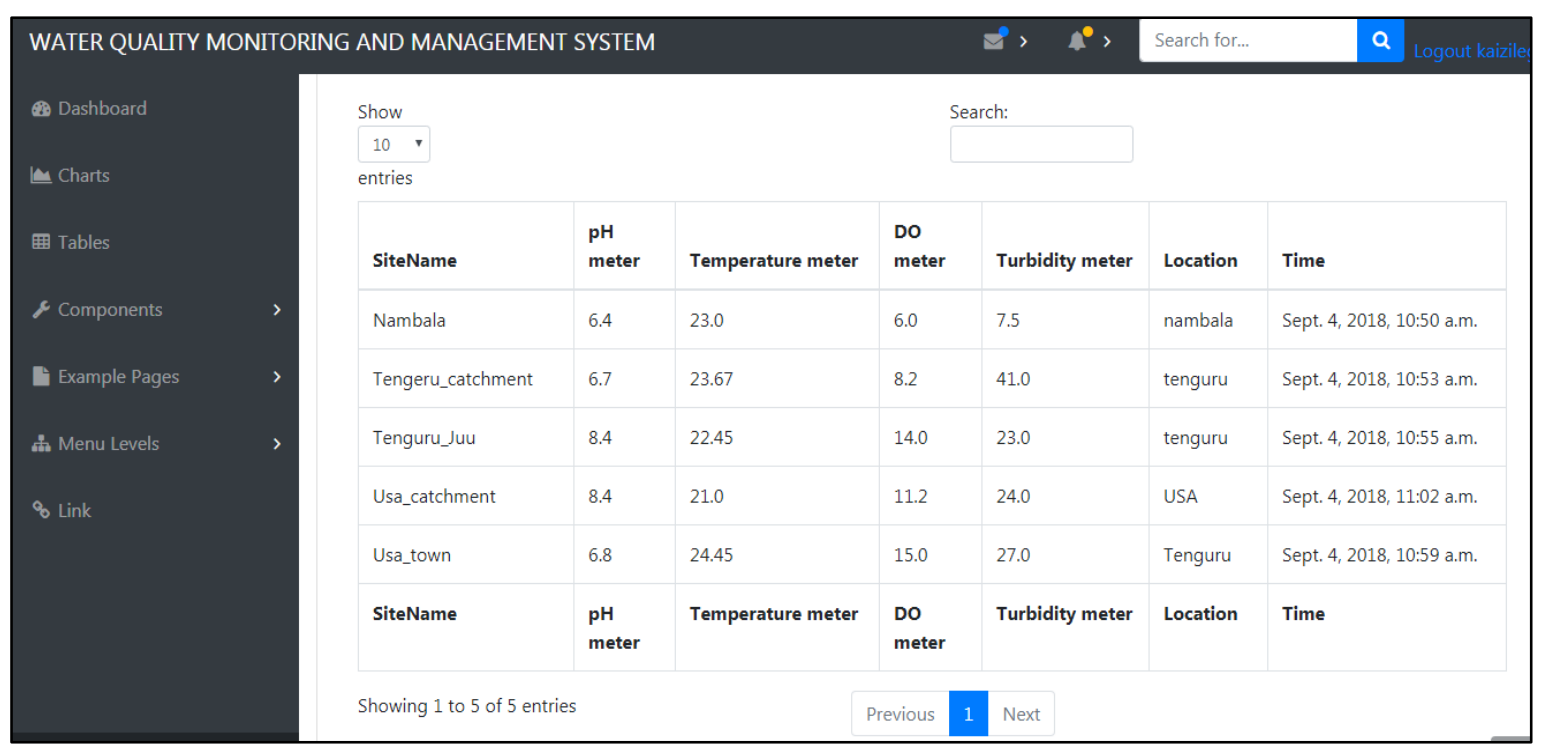

Fig.8. Sensor Measurements in form of Plaintext After AES Decryption

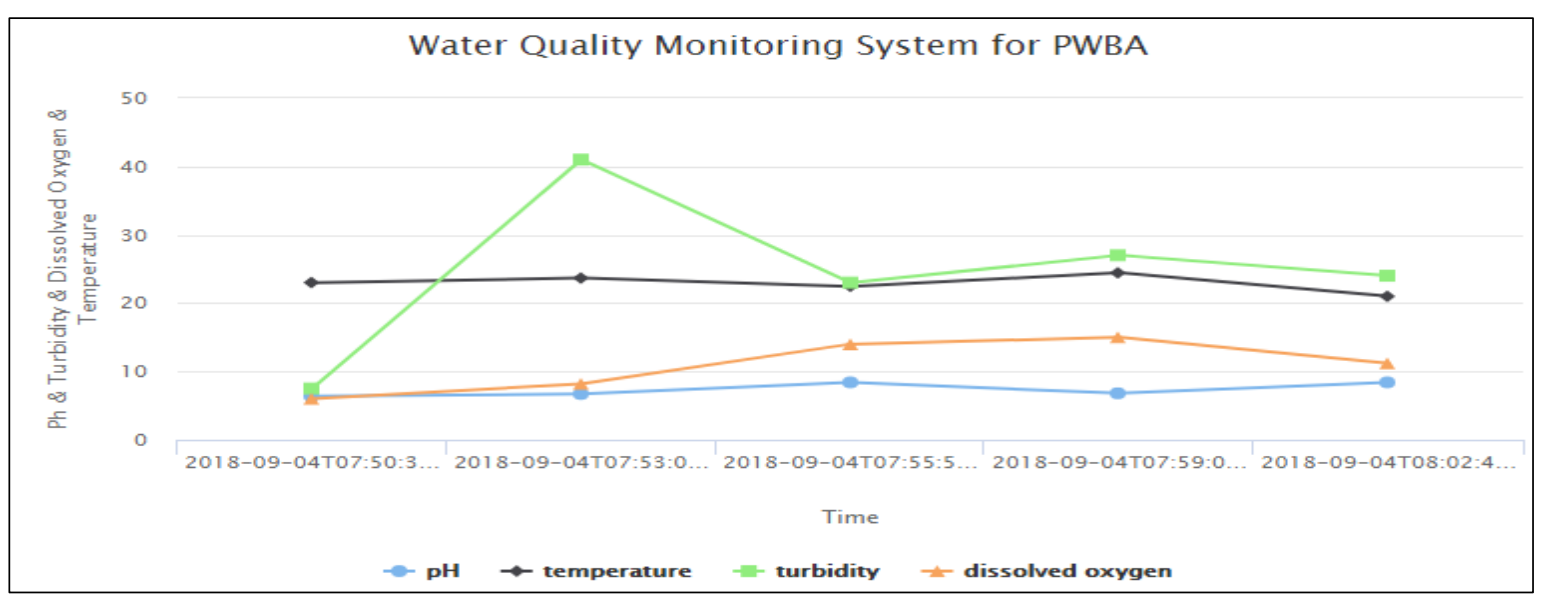

Fig.9. Sensor Measurements in Graphical Format 
Figure 10 represents the SMS sent to a site supervisor after the $\mathrm{pH}$ of 9 is measured in Nambala site. This $\mathrm{pH}$ value exceeds the standard value range (6.5-8.5) therefore trigger a notification message to be sent for intervention. Through decision support module developed in this system prototype the average values of water quality parameters ( $\mathrm{pH}$, temperature, turbidity, and dissolved oxygen) are evaluated after every hour, if the average values of water quality parameters exceed either of these ranges: $\mathrm{pH}>8.5$ AND $\mathrm{pH}<6.5$, DO $>3$ AND DO $<6$, temperature $>20$ AND temperature $<35$, and turbidity $<300$ NTU then the notification message will be sent to the responsible site supervisor.

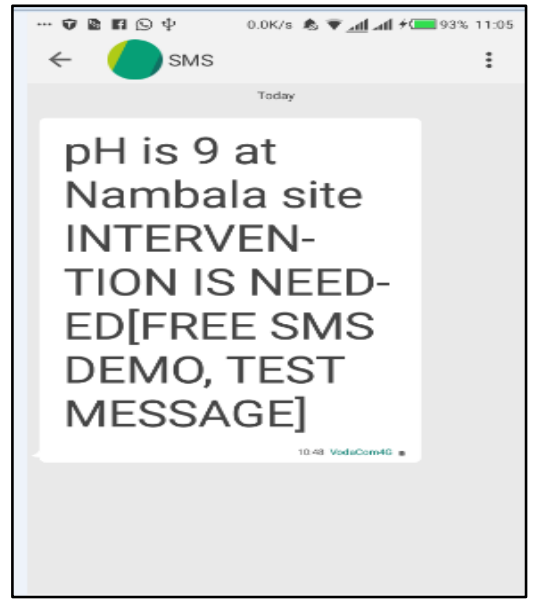

Fig.10. SMS sent to Specific Officials after $\mathrm{pH}$ value is 9 at Nambala Site.

\section{Conclusion}

In this paper, development of an innovative secured water quality monitoring system using remote sensors prototype was described. This system presents the following unique features when compared to other similar systems: it is a low cost and practically transfer sensor measurements from distant remote places to central office database using GSM/WCDMA/LTE mobile networks; the system prototype uses both battery and electrical as power sources hence reduces power limitations brought by using only battery sources; unlike others, this system prototype ensures secure transmission and storage of sensor measurements using AES algorithms. Furthermore, this system can be accessed remotely for status check and re-programming using SSH and VCN network interfaces. Moreover, this system uses a web application to provide real-time visualization of the sensor measurements in tabular and graphical formats. It also facilitates decision support by sending SMS alerts to specific officials once measured values are above predefined standard values.

\section{Reference}

[1] M. Chilundo, P. Kelderman, and J. H. Ókeeffe, "Design of a water quality monitoring network for the Limpopo River Basin in Mozambique," Phys. Chem. Earth, vol. 33, no. 8-13, pp. 655-665, 2008.

[2] G. Sotomayor, H. Hampel, and R. F. Vázquez, "Water quality assessment with emphasis in parameter optimization using pattern recognition methods and genetic algorithm," Water Res., vol. 130, pp. 353$362,2018$.

[3] A. V. Kulinkina et al., "Physicochemical parameters affecting the perception of borehole water quality 
in Ghana," Int. J. Hyg. Environ. Health, vol. 220, no. 6, pp. 990-997, 2017.

[4] UNEP, "United Nations Environment Programme The Committee of Permanent Representatives to UNEP Briefing Session - Time : 10:00 am - 12:00 pm Gigiri, UN Office at Nairobi Conference Room 4 United Nations Environment Programme 15 March 2017 The Committee of Per," no. March, pp. 10-13, 2017.

[5] Ministry of Water and Irrigation, "UNITED REPUBLIC OF TANZANIA MINISTRY OF WATER AND IRRIGATION WATER SECTOR DEVELOPMENT PROGRAMME WATER SECTOR STATUS REPORT 2016 October 2016,” Dodoma, 2016.

[6] D. V. Chapman et al., "Developments in water quality monitoring and management in large river catchments using the Danube River as an example," Environ. Sci. Policy, vol. 64, pp. 141-154, 2016.

[7] Ministry of Water, "WATER SECTOR STATUS REPORT," 2015.

[8] B. Gholamzadeh and H. Nabovati, "Concepts for Designing Low Power Wireless Sensor Network," World Acad. Sci. Eng. Technol. 45, vol. 2, no. 9, pp. 559-565, 2008.

[9] X. Hu, J. Wang, Q. Yu, W. Liu, and J. Qin, "Water Quality Monitoring System Using Zigbee Based Wireless Sensor Network," Int. J. Eng. Technol. IJET, vol. 9, pp. 24-28, 2008.

[10] N. Vijayakumar and R. Ramya, "The real-time monitoring of water quality in IoT environment," Int. Conf. Circuits, Power Comput. Technol. [ICCPCT], pp. 1-4, 2015.

[11] A. Faustine et al., "Wireless Sensor Networks for Water Quality Monitoring and Control within Lake Victoria Basin: Prototype Development Wireless Sensor Networks, Lake Victoria Basin, Gateway, Sensor Nodes," Wirel. Sens. Netw., vol. 6, no. 6, pp. 281-281, 2014.

[12] B. J. J. Voigt, "Dynamic System Development Method,” no. January, 2004.

[13] D. P. Pop and A. Altar, "Designing an MVC model for rapid web application development," Procedia Eng., vol. 69, pp. 1172-1179, 2014.

[14] J. Li and L. Li, "Comparative research on Python speed optimization strategies," Proc. - 2010 Int. Conf. Intell. Comput. Integr. Syst. ICISS2010, pp. 57-59, 2010.

[15] STMicroelectronics, "NUCLEO-F411RE," STMicroelectronics, 2018. [Online]. Available: https://www.st.com/en/evaluation-tools/nucleo-f401re.html. [Accessed: 16-Sep-2018].

[16] Hardkernel, "ODROID-XU4," Hardkernel co., Ltd., 2018. [Online]. Available: https://www.hardkernel.com/main/products/prdt_info.php. [Accessed: 15-Sep-2018].

[17] R. P. Foundation, "Raspberry Pi 3 Model B+," RASPBERRY PI FOUNDATION, 2018. [Online]. Available: https://www.raspberrypi.org/products/raspberry-pi-3-model-b-plus/. [Accessed: 02-Sep2018].

[18] DFRobot, "PH meter manual," DFRobot, 2018. [Online]. Available: https://www.dfrobot.com/product1110.html. [Accessed: 17-Sep-2018].

[19] Atlas, "EZO-Dissolved Oxygen," Atlas Scientific LLC, 2018. [Online]. Available: https://www.atlasscientific.com/_files/_datasheets/_circuit/do_EZO_datasheet.pdf. [Accessed: 15-Sep-2018].

[20] Seeed, "Grove - Temperature Sensor," Seeed Technology Co., Ltd., 2018. [Online]. Available: http://wiki.seeedstudio.com/Grove-Temperature_Sensor_V1.2/. [Accessed: 12-Sep-2018].

[21] T. Dicola, "ADS1115," Adafruit, 2018. [Online]. Available: https://learn.adafruit.com/raspberry-pianalog-to-digital-converters/ads1015-slash-ads1115. [Accessed: 31-Aug-2018].

[22] Smile, "Nition MiFi M028AT," Smile Communications Tanzania Ltd, 2018. [Online]. Available: https://smile.co.tz/product/10gb-mifi-free-sim. [Accessed: 15-Sep-2018].

[23] W. Stallings, the William Stallings Books on Computer Data and Computer Communications, Eighth Edition, vol. 139, no. 3. 2011.

[24] J. Plekhanova, "Evaluating web development frameworks: Django, Ruby on Rails and CakePHP," Inst. Bus. Inf. Technol., no. September, p. 20, 2009.

[25] D. Zhang, Z. Wei, and Y. Yang, "Research on lightweight MVC framework based on spring MVC and mybatis," Proc. - 6th Int. Symp. Comput. Intell. Des. Isc. 2013, vol. 1, pp. 350-353, 2013. 
[26] NBS, "National Environmetal Standards Compendium," 2009.

[27] P. Clark-Dickson, N. D. Pamela, A. D. Neha, and Angel, "Application-to-Person Messaging Helping enterprises to respond to consumers' changing," 2016.

\section{Authors' Profiles}

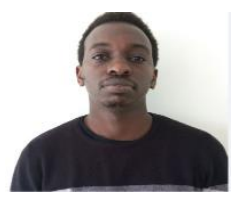

Kaizilege Mwemezi is currently a master's scholar in the Department of Communication Science and Engineering at Nelson African Institution of Science and Technology, Tanzania. He completed his Bachelor degree in Telecommunications Engineering in 2013 at the University of Dar es Salaam, Tanzania. He worked as a field engineer and received several pieces of training in the field of electronic and telecommunication.

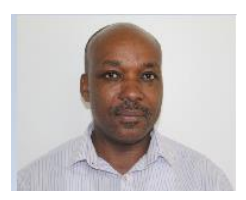

Anael E. Sam is a Senior Lecturer in the Department of Communication Science and Engineering, School of Computational and Communication Sciences and Engineering (CoCSE), at the Nelson Mandela African Institution of Science and Technology.

How to cite this paper: Kaizilege Mwemezi, Anael Sam,"Development of Innovative Secured Remote Sensor Water Quality Monitoring \& Management System: Case of Pangani Water Basin", International Journal of Engineering and Manufacturing(IJEM), Vol.9, No.1, pp.47-63, 2019.DOI: 10.5815/ijem.2019.01.05 